\title{
Single amino acid substitution in important hemoglobinopathies does not disturb molecular function and biological process
}

\author{
Viroj Wiwanitkit \\ Department of Laboratory Medicine, \\ Faculty of Medicine, Chulalongkorn \\ University, Bangkok, Thailand
}

\begin{abstract}
Hemoglobin is an important protein found in the red cells of many animals. In humans, the hemoglobin is mainly distributed in the red blood cell. Single amino acid substitution is the main pathogenesis of most hemoglobin disorders. Here, the author used a new gene ontology technology to predict the molecular function and biological process of four important hemoglobin disorders with single substitution. The four studied important abnormal hemoglobins $(\mathrm{Hb})$ with single substitution included $\mathrm{Hb} \mathrm{S}, \mathrm{Hb} \mathrm{E}, \mathrm{Hb}$, and $\mathrm{Hb}$ J-Baltimore. Using the GoFigure server, the molecular function and biological process in normal and abnormal hemoglobins was predicted. Compared with normal hemoglobin, all studied abnormal hemoglobins had the same function and biological process. This indicated that the overall function of oxygen transportation is not disturbed in the studied hemoglobin disorders. Clinical findings of oxygen depletion in abnormal hemoglobin should therefore be due to the other processes rather than genomics, proteomics, and expression levels.
\end{abstract}

Keywords: hemoglobin, amino acid, substitution, function

\section{Introduction}

Globins are an ancient and diverse superfamily of proteins. Hemoglobin is an important protein detected in red cell of many animals. In human, the hemoglobin mainly distributes in the red blood cell (Kundu et al 2003; Weber and Fago 2004). Structurally, each molecule of hemoglobin contains four globin chains and heme (Kundu et al 2003; Weber and Fago 2004). The four globin chains (two alpha chains and two beta chains) are depicted as a tetramer looking like "folded worms" (Kundu et al 2003; Weber and Fago 2004).

In the case of structurally abnormal hemoglobins, the following mechanisms can be possible: single nucleotide base substitutions leading to amino acid replacement or chain termination variants; nucleotide deletions or additions leading to deletion and frameshift variants; and nonhomologous crossing over, leading to the production of fused globin chains (Forget et al 1979). The defect of globin chain is the main disorder in hemoglobinopathies. The molecular basis of the hemoglobinopathies, disorders characterized by absent or decreased synthesis of alpha- or beta-globin chains, is quite heterogeneous (Forget et al 1979).

It is difficult to study the functional aberration due to single amino acid substitution. Luckily, the new development in molecular bioinformatics can be applied in nanoscale genomics and proteomics research. Here, the author used a new gene ontology technology to predict the molecular function and biological process of four important hemoglobin disorders with single substitution. 


\section{Materials and methods}

\section{Obtaining the sequence}

The database, Unitprot (Bairoch et al 2008), was used for data mining of the amino acid sequence for normal hemoglobin. Mutation simulating was then performed to derive each hemoglobin disorder.

\section{Prediction of molecular function and biological process}

The author performed a prediction of molecular function and biological process of normal and each abnormal hemoglobin using a novel gene ontology prediction tool namely GoFigure by Department of Computer and Information Sciences, University of Delaware, Newark, USA (Khan et al 2008). GoFigure is a computational algorithm tool which is recently developed for gene ontology study (Khan et al 2008). The tool accepts an input DNA or protein sequence, and uses the Basic Local Alignment Search Tool (BLAST) to identify homologous sequences in gene ontology annotated databases (Khan et al 2008). The purpose is to use a BLAST search to identify homologs in public databases that have been annotated with gene ontology terms (Khan et al 2008). These terms include: SwissProt, Flybase (Drosophila), the Saccharomyces Genome Database (SGD), Mouse Genome Informatics (MGI), and Wormbase (nematode) (Khan et al 2008). The contents of the results include molecular function as well as biological process of the studied protein (Khan et al 2008). Then, the predictions of molecular function and biological process were presented and compared.

\section{Results}

\section{Sequence of normal and abnormal hemoglobin}

After searching the Uniprot database, the sequence of normal hemoglobin was derived. The four studied important abnormal hemoglobins with single substitution included $\mathrm{Hb}$ $\mathrm{S}, \mathrm{Hb} \mathrm{E}, \mathrm{Hb} \mathrm{C}$, and $\mathrm{Hb}$ J-Baltimore. The details of mutation are presented in Table 1.

\section{Prediction of molecular function and biological process}

Using GoFigure server, the molecular function and biological process in normal and abnormal hemoglobin were predicted. The molecular function and biological processes of normal hemoglobin and abnormal hemoglobin
Table I Summary of the mutation of each abnormal hemoglobin

\begin{tabular}{lll}
\hline Hemoglobin (Hb) disorder & Position & Mutation \\
\hline Hb S & Beta 6 & Glu $\rightarrow$ Val \\
Hb E & Beta 26 & Glu $\rightarrow$ Lys \\
Hb C & Beta 6 & Glu $\rightarrow$ Lys \\
Hb J-Baltimore & Beta I6 & Gly $\rightarrow$ Asp \\
\hline
\end{tabular}

are similar, as presented in Figure 1. The summary of the comparison of the molecular function and biological processes between normal and abnormal hemoglobin is presented in Table 2.

\section{Discussion}

Hemoglobin research reached a renaissance in recent few years due to the discovery of globins or their genes in all living organisms and in all tissues of higher animals (Kundu et al 2003; Weber and Fago 2004). New developments brought to a re-evaluation of our understanding of the structure and function of hemoglobins (Kundu et al 2003; Weber and Fago 2004). Until now, the functional aberration due to the mutation in hemoglobin was not well demonstrated, and there is a need for improvement of our understanding on those mutated proteins' function.

Based on the recent advances in the genomics, current technologies can permit the examination of gene expression patterns of tens of thousands of genes (Bairoch et al 2008). A challenge for the biologist to interprete such data is recognizing the function of many of the hits identified in a single experiment (Khan et al 2008). While one can classically search the literature, a more rapid mean of developing some idea of potential function of a gene product is via the ontology terms that describe the gene (Khan et al 2008). The gene ontology tool is developed for this specific purpose. Many genes ontology tools have been constructed and launched for public usage. In this study, the author used a gene ontology tool to predict the function of normal and four important hemoglobin disorders with underlying single amino acid substitution.

Table 2 Summary of comparison of the molecular function and biological process of normal and abnormal hemoglobin

\begin{tabular}{lll}
\hline $\begin{array}{l}\text { Hemoglobin }(\mathbf{H b}) \\
\text { disorder }\end{array}$ & Molecular function & Biological process \\
\hline $\mathrm{Hb} \mathrm{S}$ & Oxygen transporter & Oxygen transportation \\
$\mathrm{Hb} \mathrm{E}$ & Oxygen transporter & Oxygen transportation \\
$\mathrm{Hb} \mathrm{C}$ & Oxygen transporter & Oxygen transportation \\
$\mathrm{Hb} \mathrm{J-Baltimore}$ & Oxygen transporter & Oxygen transportation \\
\hline
\end{tabular}




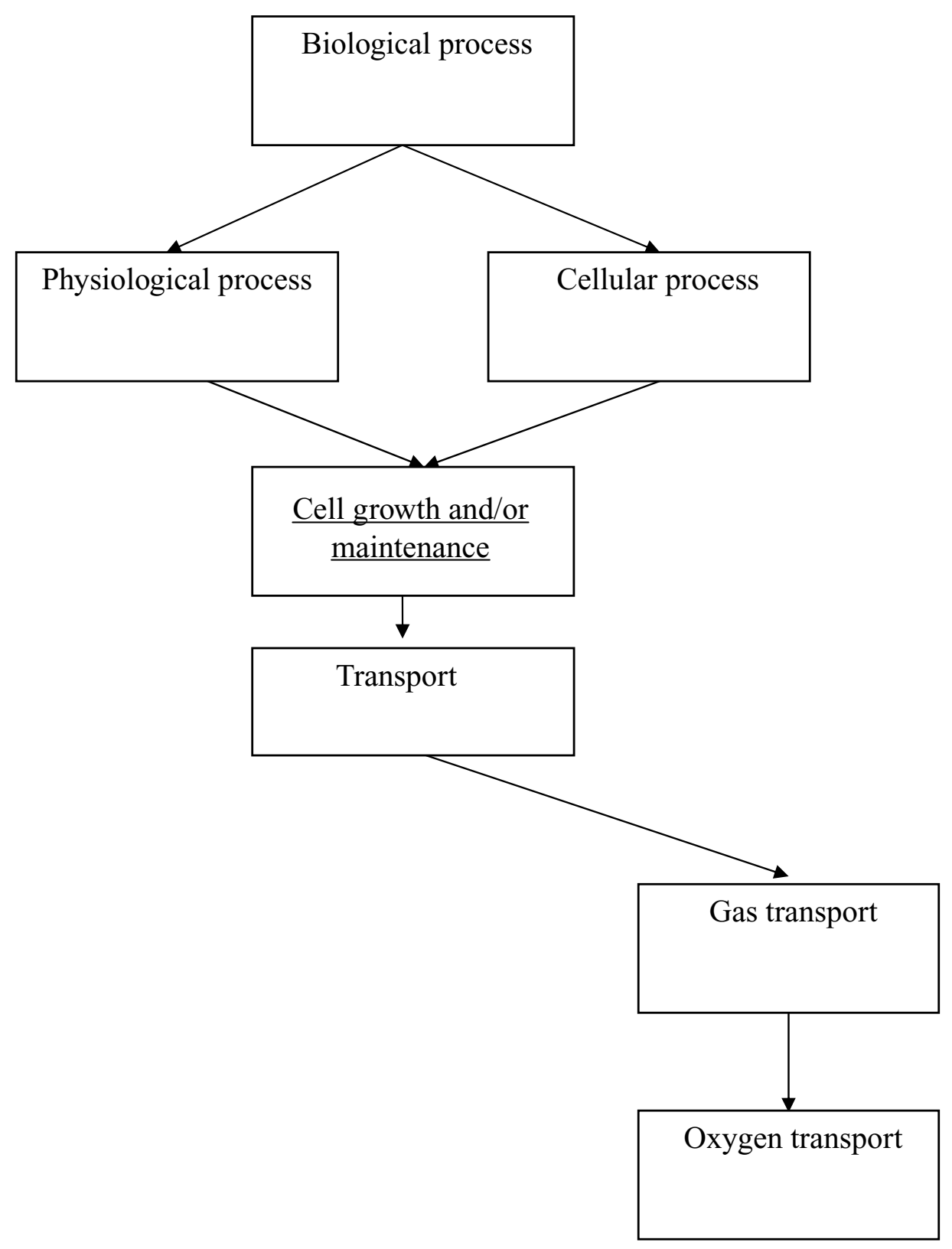

Figure I Expected biological process of normal and each abnormal hemoglobin.

Compared with normal hemoglobin, all studied abnormal hemoglobins have the same function and biological process. This indicates that the overall function of oxygen transportation is not disturbed in the studied hemoglobin disorders. This result is similar to another report by Wiwanitkit (2006) on four rare hemoglobinopathies, Hb Agrino (29Leu-->Pro), Hb Siam (15Gly -> Arg), Hb Amsterdam (32Met-->Ile) and Hb Evanston (14 Try--> Arg). Clinical findings of oxygen depletion in abnormal hemoglobins should be due to the other processes rather than genomics, proteomics and expression levels.

\section{Disclosure}

The author reports no conflict of interest.

\section{References}

Bairoch A, Apweiler R, Wu CH, et al; UniProt Consortium. 2008. The universal protein resource (UniProt). Nucleic Acids Res, 36(Database issue):D190-5.

Forget BG. 1979. Molecular genetics of human hemoglobin synthesis. Ann Intern Med, 91:605-16.

Khan S, Situ G, Decker K, et al. 2003. GoFigure: automated gene ontology annotation. Bioinformatics, 19:2484-5.

Kundu S, Trent JT 3rd, Hargrove MS. 2003. Plants, humans and hemoglobins. Trends Plant Sci, 8:387-93.

Weber RE, Fago A. 2004. Functional adaptation and its molecular basis in vertebrate hemoglobins, neuroglobins and cytoglobins. Respir Physiol Neurobiol, 144:141-59.

Wiwanitkit V. 2006. Single amino acid substitution in a-hemoglobinopathies: a study on disturbance on molecular function and biological process in some important disorders. PakJ Med Res, 45:75-7. 
